\title{
Effects of Moringa oleifera on Glycaemia and Insulin Levels: A Review of Animal and Human Studies
}

\author{
Karina Vargas-Sánchez ${ }^{1, *}$, Edwin Garay-Jaramillo ${ }^{2}$ and Rodrigo E. González-Reyes ${ }^{3, *(D)}$ \\ 1 School of Medicine, Universidad de los Andes, Bogotá 111711, Colombia \\ 2 School of Medicine, Universidad Antonio Nariño, Bogotá 110231, Colombia; edwinmedinv@gmail.com \\ 3 Universidad del Rosario, Escuela de Medicina y Ciencias de la Salud, GI en Neurociencias-NeURos, \\ Bogotá 111221, Colombia \\ * Correspondence: j.vargass@uniandes.edu.co (K.V.-S.); rodrigo.gonzalez@urosario.edu.co (R.E.G.-R.)
}

Received: 2 September 2019; Accepted: 15 October 2019; Published: 2 December 2019

\begin{abstract}
Diabetes and related neurological complications are serious worldwide public health problems. The increasing number of affected individuals make it necessary to implement novel nutritional and therapeutic interventions. The tree Moringa oleifera (MO) has been used as a food source and for traditional medicine purposes due to possible antihyperglycemic, antioxidant, anti-inflammatory, and lipid regulating properties. These properties may be explained by the presence of numerous phytochemicals in the leaves, fruits, roots and, oil of the tree. The evidence for acute antihyperglycemic effects of MO extract on diabetic animal models seems to be robust, but more chronic and long-term studies are needed. In contrast, the hypoglycemic effects of MO on humans are not as clear. The scarce number of human studies, together with a diverse range of methodologies and MO doses, may explain this. In addition, evidence regarding changes in insulin levels due to MO intervention is ambiguous, both in animal and human studies. Therefore, more structured studies are needed to clarify if $\mathrm{MO}$ has an effect on insulin levels or activity.
\end{abstract}

Keywords: Moringa oleifera; insulin; diabetes; metabolism

\section{Introduction}

Moringa oleifera (MO) (also known as "drumstick") is a tree belonging to the family Moringaceae, genus Moringa, originally native from the Himalayans but currently cultivated in many tropical and subtropical regions around the world [1]. Different parts of MO such as leaves, fruits, flowers, and roots have been used as food and in traditional medicine [2]. For example, leaves, the most commonly used part, contain many nutrients including beta-carotene; vitamins B, C, and E; minerals (calcium, iron, potassium, magnesium, etc.); essential and non-essential amino acids; and carbohydrates; among others [3]. MO has been used in traditional medicine for the treatment of various conditions and, more recently, has been proposed to be of benefit in numerous diseases including cardiovascular, diabetes, cancer, neurological, gastroenterological, and inflammatory [4]. Such broad applications attributed to MO, may be explained by the vast amounts of compounds present in the different parts of the tree. For instance, phytochemicals derived from the seeds of $\mathrm{MO}$ including glycosidic glucosinolates (GLSs), isothiocyanates (ITCs), nitriles, carbamates, and thiocarbamates, have shown anti-inflammatory, antioxidant, hypotensive, antibacterial, and chemopreventive properties [5].

Metabolic and neurological conditions have a high incidence in the general population and frequently develop into chronic complications, which severely limit quality of life. The present rise in the prevalence of diabetes, metabolic syndrome, and associated disorders (obesity and cardiovascular pathologies) prompts the need for better strategies aimed at improvement of life styles and nutrition, together with the development of more efficient therapeutic alternatives [6]. A major component 
of metabolic syndrome, hyperglycemia, is a critical factor in the development of diabetes mellitus (DM) and has been related to serious progressive damage in different organs (retina, kidneys, and nerves), as well as to the development of neurodegenerative diseases such as Alzheimer's disease [7]. DM can cause many different types of peripheral nerve injuries, the most common being a bilateral and symmetric, distal-to-proximal damage to sensory nerves in the feet (in "stocking-glove" pattern), and commonly referred to as diabetic neuropathy. It has been estimated that almost 316 million and 387 million people are affected by pre-diabetes and diabetes, respectively, worldwide, and of these people approximately 200 million develop neuropathy [8].

Different components of $\mathrm{MO}$ tree are currently under investigation in order to study possible beneficial effects on the treatment of metabolic conditions, mainly DM. It has been reported in animal models, that MO has antihyperglycemic activity [9] and can improve induced diabetic effects in rats [10]. As well, some studies in vivo and in vitro have observed some positive effects on the nervous system [11]. The main aim of this paper is to review the overall effects of MO on glycaemia and insulin, and to present relevant preclinical and clinical information regarding the use of $\mathrm{MO}$ in these aspects. Within this framework, we have focused on animal and human studies of DM, identifying this disease as a potential therapeutic target of MO.

\section{MO Tree Parts and Overall Mechanisms of Action}

\subsection{MO Fruit, Seed, and Oil}

MO seeds, and the oil derived from them, can be used for human consumption an also for commercial purposes [12]. A study on the ethanolic extract of MO seeds revealed the presence of benzyl carbamate, benzyl isothiocyanate, niazimicin, sitosterol, and niazirin [13]. The same publication reported that niazirin showed important antitumoral activities in chemical carcinogenesis. MO seeds have close to $40 \%$ oil content, among which is present a high amount of fatty acids, including oleic acid (considered a cholesterol lowering agent), tocopherols, and sterols, among others [14]. Rabbits fed with MO obtained from fruits, together with lovastatin, showed a reduction in the levels of cholesterol, phospholipids, triglycerides, very low-density lipoprotein (VLDL), low-density lipoprotein (LDL), and the atherogenic index, while high-density lipoprotein (HDL) ratio (HDL/HDL-total cholesterol) was increased [15]. In addition, MO possesses more than 30 natural antioxidants in the seeds, including kaempferol, which, apart from antioxidant functions, also improves cellular function and metabolism [16]. As mentioned previously, fruits and seeds contain GLSs, ITCs, nitriles, carbamates, and thiocarbamates, which may confer other potential uses including anti-inflammatory, antihypertensive, antifungal, antiseptic, and due to its high vitamin $C$ content, useful for scurvy prevention $[5,14]$.

\subsection{Leaves}

Due to the presence of both micro- and macronutrients, MO leaves are commonly used as a food source in many regions of the world. The leaves have a high protein content (total protein content 24.8-35.3 g/100 g) together with high levels of calcium, phosphorus, iron, and manganese [17]. Aside from its nutritional properties, MO leaves have also been used in traditional medicine for the treatment of numerous conditions. The leaves are rich in several bioactive compounds including beta-carotene, vitamins (B, C, and E), polyphenols, phenolic acids, alkaloids, GLSs, ITCs, tannins, saponins, oxalates, phytates, and antioxidants $[1,18]$. Although most studies regarding the effects of substances present in MO leaves have been conducted on animal models, the results obtained suggest a possible benefit for several human conditions. In a recent study, extracts from MO leaves showed antinociceptive and anti-inflammatory activity in a rat model of induced-arthritis [19]. A novel arabinogalactan, Moringa oleifera polysaccharide 1 (MOP-1), has been isolated from the leaves of MO showing strong antioxidant properties [20]. In addition, it has also been reported that MO leaves may be of use in viral [21] and bacterial infections [22]. In general, $\mathrm{MO}$ leaves have been reported to be of possible benefit for several chronic diseases including cardiovascular conditions, liver diseases, cancer, insulin resistance, and diabetes. For example, cardioprotective effects have been attributed to the presence of quercetin, chlorogenic acid, alkaloids, tannins, ITCs, and B-sitosterol [23]. 


\subsection{Roots and Barks}

Humans have used both $\mathrm{MO}$ roots and bark, mostly for medicinal purposes. Roots possess higher amounts of antinutrients as compared with other parts of the MO tree, limiting its edible use. Roots have higher concentrations of tannins and oxalates, which are not useful as nutritional sources; as well, they contain high levels of carbohydrates, sodium, arginine, lysine, and ascorbic acid (but they lack thiamine, riboflavin, and pyridoxine) [24]. In animal models, the use of bark and roots has proved to serve as an antiulcer agent, together with antisecretory and cytoprotective activity [25]. Other studies have reported various benefits including treatment for poor vision, joint pain, diabetes, anemia, hypertension, toothache, hemorrhoids, and uterine disorders [1].

\subsection{Mechanisms of Action of $M O$}

Each part of the MO tree provides a mix of nutrients and substances capable of producing a diverse range of effects on the organism. In this section, we will focus on the mechanism of action of the effects of $\mathrm{MO}$ extracts on metabolism, mainly on the regulation of glucose.

As mentioned above, several polyphenols are found in MO. Amongst the most important are the flavonoids quercetin and kaempferol, and the phenolic acids chlorogenic acid and caffeoylquinic acid [26]. These compounds seem to confer antihyperglycemic properties, acting as competitive inhibitors of the sodium-glucose linked transporter type 1 (SGLT1) in the mucosa of small intestine (duodenum and jejunum), thus reducing the intestinal absorption of glucose [27]. however, glucose absorption involves other mechanisms such as the glucose transporter 2 (GLUT2), which can be recruited towards small intestine basolateral membrane due to circulating glucose stimulation [28]. In DM, the capacity of the small intestine to uptake glucose is augmented, due to an increase in the expression of GLUT2 and SGLT1 [29]. This produces an extra burden on the patients suffering from $\mathrm{DM}$, further complicated by the fact that most common antidiabetic drugs such as sulfonylureas, biguanides or thiazolidinediones, have primary targets on organs other than the intestines [30]. MO has been studied as an antidiabetic agent due to its effects on the reduction of glucose levels. One of the proposed mechanisms involves quercetin, as this substance can act as an apical inhibitor of GLUT2 [31], although it has no effect on GLUT5 or SGLT1 [32]. Nevertheless, quercetin has also been shown to activate adenosine monophosphate-activated protein kinase (AMPK), to increase glucose uptake through stimulation of GLUT4 in skeletal muscle, and to decrease the production of glucose through downregulation of phosphoenolpyruvate carboxykinase (PEPCK) and glucose-6-phosphatase (G6Pase) in liver [33].

$\mathrm{MO}$ aqueous leaf extract has been shown to inhibit the activity of $\alpha$-glucosidase, pancreatic $\alpha$-amylase, and intestinal sucrose, contributing to antihyperglycemic properties [34]. These inhibitory effects are possible thanks to phenols, flavonoids, and tannins present in MO. A delay in carbohydrate digestion, caused by the inhibition of these enzymes, leads to a reduction in post-prandial hyperglycemia and hemoglobin $\mathrm{A}_{1 \mathrm{C}}\left(\mathrm{HbA}_{1 \mathrm{C}}\right)$. These inhibitory effects of flavonoids, including quercetin and kaempferol, have been biochemically explained due to an increase in the number of hydroxyl groups on the B ring, and to the presence of a 2,3-double bond [35]. In addition, these compounds have been studied regarding protective and regenerative properties on pancreatic beta-cells, augmenting insulin production and release [10]. Quercetin induces insulin secretion through phosphorylation of extracellular signal-regulated kinase 1/2 (ERK1/2) pathway together with protection of pancreatic beta-cells against oxidative damage [36].

Leaves from the tree Juglans regia, known as common walnut, also possess quercetin and kaempferol. Leaf methanolic extracts obtained from this tree were shown to inhibit the activity of protein tyrosine phosphatase 1B (PTP1B), which is in charge of the negative regulation of insulin-signaling pathway [37]. Furthermore, chlorogenic acid has been observed to enhance glucose uptake in myocytes by inhibition of PTP1B [38]. Moreover, in diabetic rats (but not in the normoglycemic animals), quercetin has been shown to significantly increase the activity of hepatic glucokinase, which mediates the conversion of glucose into glucose-6-phosphate (G6P) and is involved in insulin functional pathway [39]. Chlorogenic 
acid is also involved in hepatic gluconeogenesis as it can inhibit G6P translocase, decreasing both gluconeogenesis and glycogenolysis [40].

Hyperglycemia can lead to the formation of hydrogen peroxide and ketoaldehydes in the presence of transition metals, which hastens the production of advanced glycation-end products (AGE) [41]. AGE induce many effects including cross-linking of essential proteins, protein function alteration, and cell damage due to reactive oxygen species (ROS), together with downstream activation of proinflammatory pathways like nuclear factor $\mathrm{KB}(\mathrm{NF}-\mathrm{kB})$ [8]. A high presence of AGE and glycosylated proteins is related to the development of chronic DM complications as retinopathy, nephropathy, neuropathy, and cardiovascular conditions. Polyphenols from a MO aqueous leaf extract have been shown to inhibit protein oxidation, formation of AGE, and protein cross-linking in glycation reactions [42]. Polyphenol protection against protein glycation is believed to be possible thanks to their ability to scavenge free radicals derived from glycoxidation processes [43]. Therefore, several components of MO such as kaempferol, quercetin, chlorogenic acid, gallic acid, and ellagic acid, among others, have been studied as protective agents against ROS and free radical oxidation of DNA, proteins, and lipids [44-46]. Moreover, a recent in vitro study shown that treatment with $\mathrm{MO}$ reduces acetylation of mitochondrial proteins, increases the amount of supercomplexes and complex I activity, and decreases the activity of uncoupling protein 2 (UCP2), suggesting a possible explanation of its antihyperglycemic effects based on modulation of the mitochondrial respiratory chain [47]. The antioxidant properties of MO extract agents could also prevent the oxidation of lipids, acting as hypolipidaemic and antiatherosclerotic [48].

In addition to its antioxidant properties, extracts obtained from leaves, flowers, and seeds from MO have also exhibited anti-inflammatory effects [49-51]. These properties are mainly attributed to the presence of flavonoids, phenolic compounds, and ITCs. It has been shown that some of these compounds provide an anti-inflammatory effect, decreasing the expression of nitric oxide synthase (NOS) and inhibiting the activity of cyclooxigenase-2 (COX-2) [52]. In DM, an overproduction of mitochondrial superoxides is present in many organs, including pancreas, leading to an increase in ROS production and activation of pathogenic pathways. These ROS are responsible for oxidative stress damage to pancreatic beta-cells and insulin resistance. MO extracts reduce oxidative stress and inflammation, blocking NF- $\mathrm{kB}$ pathway activation, and thus reducing the expression of inducible nitric oxide synthase (iNOS) and other proinflammatory agents [53]. In addition, treatment with kaempferol has been shown to inhibit cellular apoptosis in pancreatic beta-cells attenuating the activity of caspase-3 and improving the antiapoptotic response trough cAMP/protein kinase A (PKA) and phosphoinositide 3-kinase (PI3K)/Akt signaling pathways [54].

MO has also been considered as a possible hypolipidemic agent. An aqueous leaf extract from $\mathrm{MO}$ was reported to have lipid-lowering properties through reduction in the formation of cholesterol micelles and inhibition of pancreatic lipase, pancreatic cholesterol esterase, and bile acid binding [34]. In addition, upregulation of the expression of peroxisome proliferator-activated receptors (PPAR) $\alpha 1$ $(\mathrm{PPAR} \alpha 1)$ and $\gamma(\mathrm{PPAR} \gamma)$ has been observed in rats which received MO seed powder [55]. PPAR are crucial for lipid metabolism, ketogenesis, and cellular energetic homeostasis, and are expressed in several organs including liver, brain, muscle, and heart, among others. As well, PPAR are involved in inflammation, immunity, and glucose regulation [56]. Thus, MO may help to modulate lipid metabolism attenuating chronic adverse effects of pathologies such as DM and atherosclerosis.

\section{Preclinical Evidence of MO Effects on Glucose and Insulin}

MO extracts have been studied for possible hypoglycemic, hypolipidemic, antioxidant, and anti-inflammatory effects. Several animal studies have evaluated MO mechanism of action and function in chronic disease models as DM. In this review, we comprised relevant investigations on animal models regarding the use of $\mathrm{MO}$ extracts on diabetes and glucose metabolism, including changes in insulin levels if reported. In total we found 20 publications, 14 studied leaves, four used seeds, one examined the fruit (pods), while another one used leaves, seeds, and stem (Table 1). Fourteen studies were conducted on rats, while five used mice and only one used both rats and mice. 
Table 1. Evidence of the effects of Moringa oleifera (MO) treatment in diabetes animal models.

\begin{tabular}{|c|c|c|c|c|c|c|}
\hline \multirow{2}{*}{ Animal Model } & \multirow{2}{*}{ MO Tree Part } & \multirow{2}{*}{ MO Treatment } & \multicolumn{3}{|c|}{ Results of MO Treatment } & \multirow{2}{*}{ Ref } \\
\hline & & & Blood Glucose & Insulin Levels & Other Effects & \\
\hline $\begin{array}{l}\text { Goto-Kakizaki (GK) } \\
\text { diabetic rats and } \\
\text { nondiabetic Wistar } \\
\text { rats used as controls }\end{array}$ & $\begin{array}{l}\text { Leaf powder (in a } \\
\text { glucose solution). }\end{array}$ & $\begin{array}{l}\text { Treatment time: } 120 \mathrm{~min} \\
\text { Con: glucose } 2 \mathrm{~g} / \mathrm{kg} \\
\text { MO: glucose } 2 \mathrm{~g} / \mathrm{kg}+200 \mathrm{mg} / \mathrm{kg} \mathrm{MO}\end{array}$ & $\begin{array}{l}\text { MO decreased BG at } 20,30,45, \text { and } 60 \mathrm{~min} \\
(p<0.05) \text { as compared with controls. }\end{array}$ & Not measured & $\begin{array}{l}\text { Food was retained for longer } \\
\text { periods in the MO treated } \\
\text { animals. }\end{array}$ & [27] \\
\hline $\begin{array}{l}\text { Alloxan-induced } \\
\text { diabetic } \\
\text { Sprague-Dawley rats }\end{array}$ & Leaf powder, & $\begin{array}{l}\text { Treatment time: } 8 \text { weeks } \\
\text { Con: Untreated } \\
\text { Con MO: } 50 \mathrm{mg} / \mathrm{kg} \text { MO } \\
\text { Diabetic Con: Untreated } \\
\text { Diabetic MO: } 50 \mathrm{mg} / \mathrm{kg} \mathrm{MO} \\
\text { Diabetic Exp: } 50 \mathrm{mg} / \mathrm{kg} \mathrm{MO} \mathrm{+} \mathrm{glibenclamide} \\
600 \mathrm{\mu g} / \mathrm{Kg}\end{array}$ & $\begin{array}{l}\text { At the second week, a significant reduction } \\
\text { was observed in } \mathrm{BG} \text { in diabetic rats treated } \\
\text { with } \mathrm{MO} \text {, from } 300 \mathrm{mg} / \mathrm{dL} \text { to } 100 \mathrm{mg} / \mathrm{dL} \text { as } \\
\text { compared with controls. }\end{array}$ & Not measured & $\begin{array}{l}\text { No change in enumeration of } \\
\text { lactic acid bacteria. }\end{array}$ & [57] \\
\hline $\begin{array}{l}\text { Wistar rats with } \\
\text { metabolic syndrome } \\
\text { (MS) induced with } \\
\text { high-fat diet }\end{array}$ & Leaf powder & $\begin{array}{l}\text { Treatment time: } 3 \text { weeks } \\
\text { Con: Untreated } \\
\text { Preventive: } 700 \mathrm{mg} / \mathrm{Kg} / \text { day MO for } 3 \text { weeks } \\
\text { before MS induction } \\
\text { Treatment: } 700 \mathrm{mg} / \mathrm{Kg} / \text { day MO for } 3 \text { weeks } \\
\text { after MS induction }\end{array}$ & $\begin{array}{l}\text { Reduction in fasting glucose levels in } \\
\text { preventive group compared with controls } \\
(80.09 \pm 5.5 \text { vs. } 103 \pm 3.8 \mathrm{mg} / \mathrm{dL}, p<0.05) \text {; } \\
\text { Reduction in OGTT in MS group compared } \\
\text { with controls }(12,616 \pm 316.8 \text { vs. } \\
138,22.5 \pm 213.93 \mathrm{mg} / \mathrm{dL} / 120 \mathrm{~min}, p<0.05) \text {. }\end{array}$ & $\begin{array}{l}\text { No changes in treated } \\
\text { rats }\end{array}$ & $\begin{array}{l}\text { Significant reduction in } \\
\text { triglyceride levels and in } \\
\text { abdominal circumference. }\end{array}$ & [58] \\
\hline $\begin{array}{l}\text { Alloxan-induced } \\
\text { diabetic mice }\end{array}$ & Leaf powder & $\begin{array}{l}\text { Treatment time: } 1,3 \text { and } 5 \mathrm{~h} \\
\text { Diabetic Con: Untreated } \\
\text { Diabetic positive Con: Insulin } 0.7 \mathrm{IU} / \mathrm{kg} \\
\text { Diabetic MO: } 100,300, \text { and } 500 \mathrm{mg} / \mathrm{kg} \text { MO }\end{array}$ & $\begin{array}{l}\text { Reduction in diabetic rats at } 5 \mathrm{~h} \text { with } 300 \\
\text { and } 500 \mathrm{mg} / \mathrm{kg} \mathrm{MO}(p<0.01) . \\
500 \mathrm{mg} / \mathrm{kg} \text { dose presented significant BG } \\
\text { reductions of } 34.3 \%, 60.9 \% \text {, and } 66.4 \% \text { after } \\
1,3 \text { and } 5 \mathrm{~h} \text {, respectively. }\end{array}$ & $\begin{array}{l}\text { No changes in } \\
\text { diabetic mice }\end{array}$ & $\begin{array}{l}\text { Significant increase in } \\
\text { catalase, no changes in } \\
\text { superoxide dismutase and } \\
\text { significant reduction in } \\
\text { MDA. }\end{array}$ & [59] \\
\hline $\begin{array}{l}\text { STZ-induced diabetic } \\
\text { Wistar rats }\end{array}$ & Aqueous leaf extract & $\begin{array}{l}\text { Treatment time: } 3 \text { weeks } \\
\text { Con: Untreated } \\
\text { Con MO: } 100,200 \text {, and } 300 \mathrm{mg} / \mathrm{kg} \mathrm{MO} \\
\text { Diabetic MO: } 100,200 \text {, and } 300 \mathrm{mg} / \mathrm{kg} \mathrm{MO} \\
\text { Diabetic positive Con: Glipizide } 2.5 \mathrm{mg} / \mathrm{kg}\end{array}$ & $\begin{array}{l}\text { Significant reduction in fasting BG of } \\
\text { diabetic rats treated with MO. Reduction } \\
\text { after 1,2, and } 3 \text { weeks with } 200 \mathrm{mg} \text { was } \\
25.9 \%, 53.5 \% \text {, and } 69.2 \% \text {, respectively. }\end{array}$ & Not measured & $\begin{array}{l}\text { Improvement of } \mathrm{Hb} \text { and total } \\
\text { protein levels. }\end{array}$ & [60] \\
\hline $\begin{array}{l}\text { Alloxan-induced } \\
\text { diabetic Wistar rats }\end{array}$ & Aqueous leaf extract & $\begin{array}{l}\text { Treatment time: } 18 \text { days } \\
\text { Con: Untreated } \\
\text { Diabetic Con: Untreated } \\
\text { Con MO: } 250 \mathrm{mg} / \mathrm{kg} \mathrm{MO} \\
\text { Diabetic MO: } 250 \mathrm{mg} / \mathrm{kg} \mathrm{MO}\end{array}$ & $\begin{array}{l}\text { Reduction in diabetic rats. MO treatment } \\
\text { reduced BG from } 400 \mathrm{mg} / \mathrm{dL} \text { to } 200 \mathrm{mg} / \mathrm{dL} \text {, } \\
p<0.05 .\end{array}$ & Not measured & $\begin{array}{l}\text { Significant reduction in } \\
\text { triglycerides and MDA. }\end{array}$ & [10] \\
\hline $\begin{array}{l}\text { STZ-induced diabetic } \\
\text { Sprague-Dawley rats }\end{array}$ & Aqueous leaf extract & $\begin{array}{l}\text { Treatment time: } 4 \text { weeks } \\
\text { Con: Untreated } \\
\text { Con MO: } 200 \mathrm{mg} / \mathrm{kg} \text { MO } \\
\text { Diabetic Con: Untreated } \\
\text { Diabetic MO: } 200 \mathrm{mg} / \mathrm{kg} \text { MO }\end{array}$ & $\begin{array}{l}\text { Reduction in BG of diabetic rats, from } \\
266.50 \pm 2.17 \mathrm{mg} / \mathrm{dL} \text { to } 148.83 \pm 2.44 \mathrm{mg} / \mathrm{dL} \\
\text { after } 200 \mathrm{mg} \text { of MO treatment, } p<0.001\end{array}$ & Not measured & $\begin{array}{l}\text { Significant rescue of GSH } \\
\text { and reduction of MDA. }\end{array}$ & [61] \\
\hline
\end{tabular}


Table 1. Cont.

\begin{tabular}{|c|c|c|c|c|c|c|}
\hline \multirow{2}{*}{ Animal Model } & \multirow{2}{*}{ MO Tree Part } & \multirow{2}{*}{ MO Treatment } & \multicolumn{3}{|c|}{ Results of MO Treatment } & \multirow{2}{*}{ Ref } \\
\hline & & & Blood Glucose & Insulin Levels & Other Effects & \\
\hline $\begin{array}{l}\text { Alloxan-induced } \\
\text { diabetic Wistar rats }\end{array}$ & Aqueous leaf extract & $\begin{array}{l}\text { Treatment time: } 18 \text { days } \\
\text { Con: Untreated } \\
\text { Diabetic Con: Untreated } \\
\text { Con MO: } 250 \mathrm{mg} / \mathrm{kg} \text { MO } \\
\text { Diabetic MO: } 250 \mathrm{mg} / \mathrm{kg} \text { MO }\end{array}$ & $\begin{array}{l}\text { Reduction in diabetic rats treated with MO. } \\
\text { Blood glucose levels lowered 3.6-fold, as } \\
\text { compared with controls, } p<0.05 \text {. }\end{array}$ & Not measured & $\begin{array}{l}\text { Normalization of SOD and } \\
\text { catalase, and significant } \\
\text { increase in GSH and } \\
\text { reduction of MDA. }\end{array}$ & [62] \\
\hline $\begin{array}{l}\text { STZ-induced diabetic } \\
\text { Wistar rats.High-fat } \\
\text { diet induced diabetes } \\
\text { C57BL/6 mice. }\end{array}$ & Aqueous leaf extract & $\begin{array}{l}\text { Treatment time: } 3 \text { weeks } \\
\text { Con: Untreated } \\
\text { Con MO: } 100 \mathrm{mg} / \mathrm{kg} \text { (rats); } \\
200 \mathrm{mg} / \mathrm{kg} \text { (mice) } \\
\text { Diabetic Con: Untreated } \\
\text { Diabetic MO: } 100 \mathrm{mg} / \mathrm{kg} \text { (rats); } 200 \mathrm{mg} / \mathrm{kg} \\
\text { (mice) } \\
\text { Diabetic positive Con: metformin } 42 \mathrm{mg} / \mathrm{kg}\end{array}$ & $\begin{array}{l}\text { Acute and chronic significant reduction in } \\
\text { diabetic rats and mice. } \\
\text { In rats, a reduction of } 53.2 \% \text { in fasting } \\
\text { glucose after } 4 \text { h of oral administration of } \\
\text { MO, whereas reduction of } 41.7 \% \text { was } \\
\text { observed after } 8 \text { h, on day } 1 \text { and day } 2, \\
p<0.05 \text {. } \\
\text { In mice, a reduction of } 34.23 \% \text { on day } 2 \text {, and } \\
58.69 \% \text { on day } 3, p<0.01 \text {. }\end{array}$ & Not measured & $\begin{array}{l}\text { Improvement of hepatic } \\
\text { functions. Significant } \\
\text { increase in HDL. Significant } \\
\text { decrease in cholesterol, } \\
\text { VLDL, LDL, and } \\
\text { triglycerides. }\end{array}$ & [63] \\
\hline $\begin{array}{l}\text { Alloxan-induced } \\
\text { diabetic Wistar rats }\end{array}$ & $\begin{array}{l}\text { Methanolic leaf } \\
\text { extract }\end{array}$ & $\begin{array}{l}\text { Treatment time: } 6 \text { weeks } \\
\text { Con: Untreated } \\
\text { Diabetic Con: Untreated } \\
\text { Diabetic MO: } 300 \text { or } 600 \mathrm{mg} / \mathrm{kg} \mathrm{MO} \\
\text { Diabetic positive Con: metformin } 100 \mathrm{mg} / \mathrm{kg}\end{array}$ & $\begin{array}{l}\text { Reduction in diabetic rats. BG was reduced } \\
\text { by } 76 \% \text { at } 300 \mathrm{mg} / \mathrm{kg} \text { and } 84 \% \text { at } 600 \mathrm{mg} / \mathrm{kg} \text {, } \\
p<0.001 . \mathrm{In} \text { addition, glucose tolerance was } \\
\text { improved by } 56 \% \text { and } 57 \% \text { with } 300 \text { or } \\
600 \mathrm{mg} / \mathrm{kg} \text { of } \mathrm{MO} \text {, respectively, } p<0.001 \text {. }\end{array}$ & $\begin{array}{l}\text { Significant increase in } \\
\text { diabetic rats. Serum } \\
\text { insulin levels } \\
\text { increased } 1.3-1.7 \text {-fold, } \\
p<0.01 \text {. }\end{array}$ & $\begin{array}{l}\text { Significant reductions in } \\
\text { triglycerides, total cholesterol } \\
\text { and LDL. Significant increase } \\
\text { in HDL. }\end{array}$ & [64] \\
\hline $\begin{array}{l}\text { STZ-induced diabetic } \\
\text { Wistar rats }\end{array}$ & $\begin{array}{l}\text { Methanolic leaf } \\
\text { extract }\end{array}$ & $\begin{array}{l}\text { Treatment time: } 6 \text { weeks } \\
\text { Con: Untreated } \\
\text { Con MO: } 250 \mathrm{mg} / \mathrm{kg} \text { MO } \\
\text { Diabetic Con: Untreated } \\
\text { Diabetic MO: } 250 \mathrm{mg} / \mathrm{kg} \text { MO }\end{array}$ & $\begin{array}{l}\text { Reduction in diabetic rats from } 30.96 \text { to } \\
27.6 \mathrm{mmol} / \mathrm{L}, p<0.05 \text {. }\end{array}$ & Not measured & $\begin{array}{l}\text { Reduction in the activities of } \\
\text { hepatic enzymes. Significant } \\
\text { reduction of cholesterol, LDL, } \\
\text { IL-6, TNF and MCP-1. } \\
\text { Significant increase in HDL. }\end{array}$ & [65] \\
\hline $\begin{array}{l}\text { STZ-induced diabetic } \\
\text { Wistar rats }\end{array}$ & $\begin{array}{l}\text { Methanolic leaf } \\
\text { extract }\end{array}$ & $\begin{array}{l}\text { Treatment time: } 3 \text { weeks } \\
\text { Con: Untreated } \\
\text { Diabetic Con: Untreated } \\
\text { Diabetic MO: } 200 \mathrm{mg} / \mathrm{kg} / \text { day MO }\end{array}$ & $\begin{array}{l}\text { Reduction in BG levels in diabetic rats from } \\
229 \pm 9.05 \mathrm{mg} / \mathrm{dL} \text { to } 86 \pm 4.2 \mathrm{mg} / \mathrm{dL}, p<0.05\end{array}$ & Not measured & $\begin{array}{l}\text { Oxidative stress attenuation } \\
\text { and normalization of } \\
\text { mitochondrial function } \\
\text { in liver. }\end{array}$ & [66] \\
\hline $\begin{array}{l}\text { Alloxan-induced } \\
\text { diabetic Wistar rats }\end{array}$ & Ethanolic leaf extract & $\begin{array}{l}\text { Treatment time: } 3 \text { weeks } \\
\text { Con: Untreated } \\
\text { Diabetic Con: Untreated } \\
\text { Diabetic positive Con: Sitagliptin } 50 \mathrm{mg} / \mathrm{kg} \\
\text { Diabetic MO: } 300 \mathrm{mg} / \mathrm{kg} \mathrm{MO} \\
\text { Sitagliptin + MO: } 50 \mathrm{mg} / \mathrm{kg}+300 \mathrm{mg} / \mathrm{kg} \mathrm{MO}\end{array}$ & $\begin{array}{l}\text { Acute but not chronic significant reduction } \\
\text { in diabetic rats. } \\
\text { The co-administration of sitagliptin and MO } \\
\text { produced a decrease of } 60 \% \\
(90.00 \pm 9.77 \mathrm{mg} / \mathrm{dL}, p<0.01) \text { in fasting BG } \\
\text { after } 2 \text { weeks, as compared with day } 1 \text { levels } \\
\text { ( } 226.85 \pm 21.81 \mathrm{mg} / \mathrm{dl}) \text {. No significant } \\
\text { reduction at } 4 \mathrm{weeks}(38 \% \text {, } \\
138.57 \pm 15.66 \mathrm{mg} / \mathrm{dL}) \text {. }\end{array}$ & $\begin{array}{l}\text { No changes in } \\
\text { diabetic rats }\end{array}$ & $\begin{array}{l}\text { No changes in } \\
\text { hyperglycemic retinopathy. }\end{array}$ & [67] \\
\hline
\end{tabular}


Table 1. Cont.

\begin{tabular}{|c|c|c|c|c|c|c|}
\hline \multirow{2}{*}{ Animal Model } & \multirow{2}{*}{ MO Tree Part } & \multirow{2}{*}{ MO Treatment } & \multicolumn{3}{|c|}{ Results of MO Treatment } & \multirow{2}{*}{ Ref } \\
\hline & & & Blood Glucose & Insulin Levels & Other Effects & \\
\hline $\begin{array}{l}\text { STZ-induced diabetic } \\
\text { Long Evan rats }\end{array}$ & Ethanolic leaf extract & $\begin{array}{l}\text { Treatment time: } 120 \mathrm{~min} \\
\text { Diabetic Con: Untreated } \\
\text { Diabetic MO: } 250 \mathrm{mg} / \mathrm{kg} \text { MO } \\
\text { Diabetic positive Con: Glibenclamide } \\
0.5 \mathrm{mg} / \mathrm{kg}\end{array}$ & $\begin{array}{l}\text { Reduction in diabetic rats (from } \sim 6.5 \text { to } \\
\sim 5.5 \mathrm{mmol} / \mathrm{L}, p<0.05 \text { ). }\end{array}$ & $\begin{array}{l}\text { No changes in } \\
\text { diabetic rats }\end{array}$ & - & [9] \\
\hline $\begin{array}{l}\text { C57BLKS/J } \\
\text { Iar-+Leprdb/+Ledpr }{ }^{\mathrm{db}} \\
\text { and C57BLKS/J } \\
\text { Iar-m+/Lepr }{ }^{\mathrm{db}} \text { mice }\end{array}$ & $\begin{array}{l}\text { Separate ethanolic } \\
\text { extracts from leaves, } \\
\text { seeds, and stem }\end{array}$ & $\begin{array}{l}\text { Treatment time: } 5 \text { weeks } \\
\text { Con: Untreated } \\
\text { MO: } 150 \mathrm{mg} / \mathrm{kg} \mathrm{MO} \\
\text { Metformin: } 150 \mathrm{mg} / \mathrm{kg}\end{array}$ & $\begin{array}{l}\text { Reduction in diabetic mice (only studied in } \\
\text { leaves extract). Reduction in fasting BG } \\
\text { from } 483 \text { to } 312 \mathrm{mg} / \mathrm{dL}, p<0.05 \text {. }\end{array}$ & $\begin{array}{l}\text { Significant increase in } \\
\text { diabetic mice (only } \\
\text { studied in leaves } \\
\text { extract). Increased } \\
\text { insulin levels from } \\
946 \pm 92 \text { to } 1678 \pm 268 \\
\text { pg/mL, } p<0.05 \text {. }\end{array}$ & $\begin{array}{l}\text { Significant decrease in } \\
\text { triglycerides and LDL. } \\
\text { Decreased expression of } \\
\text { inflammatory markers in the } \\
\text { kidneys. }\end{array}$ & {$[68]$} \\
\hline $\begin{array}{l}\text { STZ-induced diabetic } \\
\text { Albino rats }\end{array}$ & Seed powder & $\begin{array}{l}\text { Treatment time: } 4 \text { weeks } \\
\text { Con: Untreated } \\
\text { Diabetic Con: Untreated } \\
\text { Diabetic MO: } 50 \text { or } 100 \mathrm{mg} / \mathrm{kg} \text { MO for } 4 \\
\text { weeks }\end{array}$ & $\begin{array}{l}\text { Reduction in diabetic rats from } 266 \text { to } 148 \\
\mathrm{mg} / \mathrm{dL}, p<0.05\end{array}$ & Not measured & $\begin{array}{l}\text { Significant decrease in } \\
\text { HbA } A_{1 C} \text {. Significant reduction } \\
\text { in lipid peroxide. Significant } \\
\text { increase in antioxidant } \\
\text { enzymes. Significant } \\
\text { decrease in IL-6. } \\
\text { Improvement of urinary and } \\
\text { kidney functions. }\end{array}$ & [69] \\
\hline $\begin{array}{l}\text { STZ-induced diabetic } \\
\text { ICR mice }\end{array}$ & $\begin{array}{l}\text { Compounds extracted } \\
\text { from seeds }\end{array}$ & $\begin{array}{l}\text { Treatment time } 2 \text { weeks } \\
\text { Con: Untreated } \\
\text { Diabetic Con: Untreated } \\
\text { Diabetic MO: } 20 \mathrm{mg} / \mathrm{kg} \text { per MO compound }\end{array}$ & Reduction in diabetic mice $(p<0.05)$ & Not measured & - & [71] \\
\hline
\end{tabular}


Table 1. Cont.

\begin{tabular}{|c|c|c|c|c|c|c|}
\hline \multirow{2}{*}{ Animal Model } & \multirow{2}{*}{ MO Tree Part } & \multirow{2}{*}{ MO Treatment } & \multicolumn{3}{|c|}{ Results of MO Treatment } & \multirow{2}{*}{ Ref } \\
\hline & & & Blood Glucose & Insulin Levels & Other Effects & \\
\hline $\begin{array}{l}\text { Alloxan-induced } \\
\text { Swiss-Webster mice }\end{array}$ & $\begin{array}{l}\text { N-hexane seeds } \\
\text { extract }\end{array}$ & $\begin{array}{l}\text { Treatment time: } 8 \text { days } \\
\text { Con: Untreated } \\
\text { Con MO: } 40,60 \text {, and } 80 \mathrm{mg} / \mathrm{kg} \mathrm{MO} \\
\text { Diabetic MO: } 40,60 \text {, and } 80 \mathrm{mg} / \mathrm{kg} \mathrm{MO} \\
\text { Diabetic beta-sitosterol: } 18,25 \text {, and } 35 \mathrm{mg} / \mathrm{kg}\end{array}$ & $\begin{array}{l}\text { Significant reduction in diabetic mice (both } \\
\text { in acute }<6 \mathrm{~h} \text {, and in subchronic treatment, } \\
\text { up to } 8 \text { days). } \\
\text { MO doses of } 40,60 \text {, and } 80 \mathrm{mg} / \mathrm{kg} \text {, shown a } \\
\text { decrease of BG after } 6 \mathrm{~h} \text { of } 49.2 \%(\sim 130 \\
\mathrm{mg} / \mathrm{dL}, p<0.05), 53.4 \%(\sim 115 \mathrm{mg} / \mathrm{dL}, p< \\
0.05), \text { and } 60.6 \%(\sim 100 \mathrm{mg} / \mathrm{dL}, p<0.05) \text {, } \\
\text { respectively, as compared with controls } \\
(\sim 240 \mathrm{mg} / \mathrm{dL}) \text {. } \\
\text { Treatment with } \mathrm{MO} \text { at day } 8 \text { showed BG } \\
\text { reduction of } 55.1 \%(\sim 110 \mathrm{mg} / \mathrm{dL}, p<0.05) \\
\text { with } 40 \mathrm{mg} / \mathrm{kg}, 62.0 \%(\sim 100 \mathrm{mg} / \mathrm{dL}) \text { with } 60 \\
\mathrm{mg} / \mathrm{kg}, \text { and } 70.1 \%(\sim 70 \mathrm{mg} / \mathrm{dL}, p<0.05) \\
\text { with } 80 \mathrm{mg} / \mathrm{kg} \text {, as compared with controls } \\
(\sim 250 \mathrm{mg} / \mathrm{dL}) \text {. }\end{array}$ & $\begin{array}{l}\text { Significant increase in } \\
\text { diabetic mice } \\
\text { ( } 8 \text { weeks after } \\
\text { subchronic MO } \\
\text { treatment). Treatment } \\
\text { with } 40 \mathrm{mg} / \mathrm{kg} \\
\text { showed an increase to } \\
\sim 2.7 \mathrm{mg} / \mathrm{L}(p<0.05) \text {, } \\
\text { with } 60 \mathrm{mg} / \mathrm{kg} \text { to } \\
\sim 3 \mathrm{mg} / \mathrm{L}(p<0.05) \text {, } \\
\text { and with } 80 \mathrm{mg} / \mathrm{kg} \text { to } \\
\sim 3.7 \mathrm{mg} / \mathrm{L}(p<0.05), \\
\text { compared with } \\
\text { controls }(1.5 \mathrm{mg} / \mathrm{L}) .\end{array}$ & $\begin{array}{l}\text { Inhibition of } \\
\text { alpha-glucosidase activity, } \\
\text { increase in antioxidant } \\
\text { activity, and reduction in } \\
\mathrm{HbA}_{1 \mathrm{C}} \text { levels. } \\
\text { Improvement of diabetic } \\
\text { neuropathy with significant } \\
\text { decrease in tail-flick and hot } \\
\text { plate latencies, and } \\
\text { significant attenuation of } \\
\text { static mechanical allodynia. }\end{array}$ & [72] \\
\hline $\begin{array}{l}\text { STZ-induced diabetic } \\
\text { Wistar rats }\end{array}$ & $\begin{array}{l}\text { Methanolic fruit } \\
\text { extract }\end{array}$ & $\begin{array}{l}\text { Treatment time: } 3 \text { weeks } \\
\text { Con: Untreated } \\
\text { Diabetic Con: Untreated } \\
\text { Con + MO: } 150 \text { or } 300 \mathrm{mg} / \mathrm{kg} \mathrm{MO} \\
\text { Diabetic + MO: } 150 \text { or } 300 \mathrm{mg} / \mathrm{kg} \text { MO } \\
\text { Diabetic positive Con: Glibenclamide } \\
0.3 \mathrm{mg} / \mathrm{kg}\end{array}$ & $\begin{array}{l}\text { Reduction in diabetic rats treated with both } \\
150 \mathrm{mg} / \mathrm{kg} \mathrm{MO}(261.28 \pm 13.20 \text { to } 213.37 \pm \\
28.36 \mathrm{mg} / \mathrm{dL}, p<0.05) \mathrm{and} 300 \mathrm{mg} / \mathrm{kg} \mathrm{MO} \\
(261.8 \pm 13.20 \text { to } 162.37 \pm 22.78 \mathrm{mg} / \mathrm{dL} \\
p<0.05) .\end{array}$ & 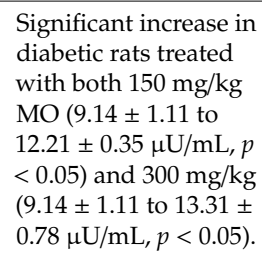 & $\begin{array}{l}\text { Increases in protein levels, } \\
\text { SOD, GSH, and catalase } \\
\text { activity. Significant decrease } \\
\text { in lipid peroxidation }\end{array}$ & [73] \\
\hline
\end{tabular}

Abbreviations: Blood glucose (BG); control (Con); experimental (Exp); glutathione (GSH); hemoglobin (Hb); high-density lipoprotein (HDL); low-density lipoprotein (LDL); malondialdehyde (MDA); metabolic syndrome (MS); monocyte chemoattractant protein-1 (MCP-1); oral glucose tolerance test (OGTT); superoxide dismutase (SOD); tumor necrosis factor (TNF); very low-density lipoprotein (VLDL). 
Glucose intolerance has been improved in a study using diabetic male Goto-Kakizaki rats and nondiabetic male Wistar rats treated with MO leaf extract [27]. A significant reduction in glycaemia at 60 and $120 \mathrm{~min}$ after glucose administration was found in Goto-Kakizaki rats as opposed to control Wistar rats, which had a nonsignificant reduction. A study performed on male Wistar rats with diabetes induced by streptozotocin (STZ) intraperitoneal injection (i.p.) $(55 \mathrm{mg} / \mathrm{kg})$, showed that treatment with $\mathrm{MO}$ aqueous leaf extract at different doses improved glucose tolerance in diabetic rats, as well as reducing glycaemia in controls, and normalizing glycaemia in sub, mild, and severely diabetic rats [60]. The same study used glipizide (a sulfonylurea used to treat DM in humans) as a positive control, finding that the MO extract produced better results than this drug. In addition, the study also observed an improvement in hemoglobin and total protein levels in MO-treated animals and absence of proteins and glucose in urine. Wistar rats of both sexes with diabetes induced after STZ injection $(50 \mathrm{mg} / \mathrm{kg}$, i.p.), were treated with a methanolic extract from MO fruits [73]. The authors showed that diabetic animals treated with MO presented a reduction in blood glucose and nitric oxide, together with an increase in serum insulin, protein levels, and improved antioxidant function. The same study used glibenclamide (a sulfonylurea commonly used to treat DM in humans) as a positive control, and obtained similar results with the animals treated with MO. A similar study with STZ-induced $(90 \mathrm{mg} / \mathrm{kg}$, i.p.) diabetes in Long Evan rats showed better performance of MO ethanolic leaf extract as compared with glibenclamide, although MO-treated diabetic animals showed no changes in insulin levels [9]. A recent publication reproduced these results obtaining comparable results from MO-treated diabetic Sprague-Dawley rats and glibenclamide-treated diabetic Sprague-Dawley rats [57].

Female alloxan-induced $(100 \mathrm{mg} / \mathrm{kg}$, i.p.) diabetic Wistar rats treated with $\mathrm{MO}$ aqueous leaf extract, had a significant glycaemia reduction, returning to normal levels, together with improvement of lipid metabolism [10]. Furthermore, treatment with MO provided evidence of hepatocyte and pancreatic beta-cell regeneration. A significant glycaemia reduction was observed in STZ-induced (60 mg/kg, i.p.) diabetes male Sprague-Dawley rats treated with MO aqueous leaf extract [61]. This study also showed that MO treatment preserved the histology of beta-cells, increased glutathione (GSH), and reduced malondialdehyde (MDA). Rats with STZ-induced diabetes $(60 \mathrm{mg} / \mathrm{kg}$ intravenous (i.v.)), were treated with either 50 or $100 \mathrm{mg} / \mathrm{kg}$ of MO seed powder, finding a significant reduction in glycaemia, but failing to return to control levels [69]. In addition, the authors reported that treatment with MO improved various factors including lipid peroxidation, antioxidant enzymes (MDA, catalase, superoxide dismutase (SOD), and GSH), immunoglobulins (decreased IgG and IgA), anti-inflammatory (decreased IL-6), $\mathrm{HbA}_{1 \mathrm{C}}$, electrolytes (sodium and potassium), and urinary and kidney functions (creatinine, blood urea nitrogen (BUN), and uric acid). Although both treatments with MO (50 and $100 \mathrm{mg} / \mathrm{kg}$ ) significantly improved the previously mentioned factors, the dose of $100 \mathrm{mg} / \mathrm{kg}$ was more effective. Similar antioxidant and hepatoprotective results were observed in other alloxan-induced and STZ-induced diabetes rat models $[62,65]$. In addition to its antihyperglycemic function, MO extract have also shown mitochondrial protective effects. Treatment with MO methanolic leaf extract on STZ-induced ( $55 \mathrm{mg} / \mathrm{kg}$, i.p.) diabetic Wistar rats, showed elevation of heme oxygenase- 1 and GSH levels and a reduction in ROS formation and in lipoperoxidation in liver mitochondria [66]. Another study, using male Wistar rats with alloxan-induced diabetes $(120 \mathrm{mg} / \mathrm{kg}$, i.p.) and treated with MO methanolic leaf extract, showed a significant reduction in glycaemia and a significant increase in insulin levels as compared with non-treated diabetic animals [64]. The authors found that treatment with MO produced similar results to treatment with metformin (a biguanide frequently used to treat DM in humans). Furthermore, the same study observed a significant reduction in blood total cholesterol, LDL, and triglycerides, and a significant increase in HDL. The effects of MO have also been studied in diabetic transgenic mice models. Tang et al. [68] treated C57BLKS/J Iar-+Lepr ${ }^{\mathrm{db}} /+$ Ledpr ${ }^{\mathrm{db}}$ and C57BLKS/J Iar-m+/Lepr ${ }^{\mathrm{db}}$ mice with a MO ethanolic extract obtained from leaves, seeds, and stem, and found a significant decrease in blood glucose concentration from day 14 to day 35, as well as a significant increase in insulin levels and a significant decrease in triglycerides and LDL. The ethanolic 
leaves extract showed the strongest antioxidant effects as compared with seeds and stem, but glycemic effects were only studied on leaves.

Olurishe et al. [67] showed that chronic administration (up to 42 days) of MO ethanolic leaf extract together with sitagliptin (an oral antihyperglycemic used in DM type 2) were useful for glycemic reduction in alloxan-induced $(150 \mathrm{mg} / \mathrm{kg}$, i.p.) diabetic Wistar rats, although administration of MO alone only produced a significant reduction in fasting blood glucose levels on day 21, failing to offer a significant reduction at later times of the study. Furthermore, the same paper reported no significant reduction in insulin levels with MO extract treatment, as well as no changes in hyperglycemic induced pathologic lesions in the retina. A related study by the same group, also using alloxan-induced diabetic Wistar rats treated with sitagliptin and MO ethanolic leaf extract, reported no changes in the levels of blood electrolytes or albumin (glycaemia or insulin were not measured), a significant increase in urea levels and triglycerides, and no benefit over kidney function or structure [74]. A recent study done in STZ-induced ( $45 \mathrm{mg} / \mathrm{kg}$, i.p.) diabetic female Wistar rats and in high-fat diet induced diabetes female C57BL/6 mice, showed both acute and chronic significant reduction in glycaemia in the animals treated with MO aqueous leaf extract [63]. The chronic effects were only measured up to day 22, so it is unknown if this study could have shown similar results to those by Olurishe et al. [67] that measured glycaemia up to 42 days. In addition, both diabetic rats and mice treated with MO presented improvement of hepatic functions, a significant reduction in total cholesterol, triglycerides, LDL, and VLDL, and a significant increase in HDL. The MO aqueous leaf extract effects were more effective on the diabetic mice model than the rat diabetes model. A study that induced obesity and insulin resistance after feeding five-week-old C57BL/6J mice with a very-high-fat diet, showed MO treatment improved glucose tolerance [70]. Animals were given isothiocyanate-enriched $\mathrm{MO}$ seed treatment mixed with either very-high-fat or low-fat diets ad libitum up to 12 weeks. The study reported that MO-treated mice had reduced blood glucose as compared with control (very-high-fat diet without MO treatment) mice, during the 12 weeks. The low-fat diet animals only showed a blood glucose reduction during week nine of the study. In addition, the authors reported other benefits of the MO treatment, such as anti-inflammatory, antioxidant, and modulation of gut microbiome.

Specific compounds from the MO tree, including $\mathrm{N}, \mathrm{N}^{\prime}$-bis $\{4-[(\alpha$-L-rhamnosyloxy $)$ benzyl $]\}$ thiourea, S-Methyl-N-\{4-[( $\alpha$-L-rhamnosyloxy)benzyl] $\}$ thiocarbamate, and niazirinA, have also been studied for possible antihyperglycemic and antidiabetic effects in preclinical experiments. A recent study found that the above compounds, obtained from $\mathrm{MO}$ seeds, significantly reduced blood glucose levels in STZ-induced ( $40 \mathrm{mg} / \mathrm{kg}$ ) diabetic Institute of Cancer Research (ICR) mice [71]. A protein isolate obtained from MO leaves (57.5\% of the soluble protein of the leaf extract) was studied in alloxan-induced $(150 \mathrm{mg} / \mathrm{kg}$, i.p.) male diabetic mice [59]. A single i.p. dose of the protein isolate at 300 and $500 \mathrm{mg} / \mathrm{kg}$ (but not with $100 \mathrm{mg} / \mathrm{kg}$ ) was able to induce a significant hypoglycemic reaction in the diabetic mice, although, after heating the protein isolate at $98^{\circ} \mathrm{C}$ or after oral administration, the effect was abolished. This protein isolate failed to alter insulin or SOD levels, but significantly increased catalase and reduced MDA levels.

A metabolic syndrome model induced through a high-fat diet in Wistar rats showed that a three week MO leaf powder treatment $(700 \mathrm{mg} / \mathrm{Kg}$ once per day) was able to improve results on the oral glucose tolerance test, together with a decrease in triglyceride levels and in abdominal circumference [58]. No changes were observed in insulin tolerance test, total cholesterol, blood pressure, or fasting glucose.

The previous investigations showed a hypoglycemic effect of $\mathrm{MO}$ in different rodent models under various conditions. Despite the use of a very wide range of MO doses, strongest antihyperglycemic effects on diabetic animals were mostly reported between 200 and $300 \mathrm{mg} / \mathrm{kg}$. The study by Wang et al. [71], showed significant hypoglycemic effect with a low value of $20 \mathrm{mg} / \mathrm{kg}$, although this result may be explained as this study used isolated MO compounds, instead of tree parts, so the doses are not comparable. As mentioned previously, several components of MO may explain these effects on 
glycemia. For example, the flavonoid kaempferol has been shown to improve glycolysis, glucose uptake, glycogen synthesis, AMP-activated protein kinase (AMPK) activity, and GLUT-4 expression [58,75].

Most papers found, failed to report effects of MO on insulin levels. As a critical component of DM, future studies should evaluate insulin levels together with glycaemia levels in order to determine an overall effect of MO on this disease. Evidence for insulin-modifying effects of MO is still under scrutiny. The publications of Gupta et al. [73], Olayaki et al. [64], and Tang et al. [68], reported an increase in insulin in diabetic rodent models treated with $\mathrm{MO}$ methanolic fruit, methanolic leaf, and ethanolic leaf extracts, respectively. While a decrease in insulin levels was reported in a Wistar rat model for polycystic ovary syndrome treated with $\mathrm{MO}$ aqueous leaf extract, although this last study failed to measure glycemic levels [76]. Furthermore, no changes in insulin were reported in the works by Azad et al. [9], Olurishe et al. [67], Paula et al. [59], and López et al. [58]. These variations in obtained results regarding insulin may be explained by the diverse experimental conditions in the studies, where tree parts, animal model, and MO doses were different (Table 1).

\section{Clinical Evidence of MO Effects on Glucose and Insulin}

Despite the number of in vitro and animal studies reporting the antihyperglycemic effects of MO, few clinical trials or studies have been conducted in humans. In our search, we found six published studies, which measured the effects of MO on blood glucose levels in humans (Table 2). 
Table 2. Evidence of the effects of MO treatment in human studies.

\begin{tabular}{|c|c|c|c|c|c|c|}
\hline \multirow{2}{*}{ Condition (Type of Study) } & \multirow{2}{*}{ Participants } & \multirow{2}{*}{ MO Tree Part } & \multirow{2}{*}{$\begin{array}{l}\text { MO Treatment } \\
\text { (Duration) }\end{array}$} & \multicolumn{2}{|c|}{ Results of MO Treatment } & \multirow{2}{*}{ Ref } \\
\hline & & & & Blood Glucose & Other Effects & \\
\hline $\begin{array}{l}\text { Postmenopausal women } \\
\text { (Randomized controlled trial) }\end{array}$ & $\begin{array}{l}30 \text { females; } \\
\text { age range } 45-55 \text { years }\end{array}$ & Leaf powder & $7 \mathrm{~g}$ daily for 3 months & $\begin{array}{l}\text { Reduction from a baseline of } 125.6 \pm \\
9.15 \text { to } 106.7 \pm 7.23 \mathrm{mg} / \mathrm{dL}(p \leq 0.01) \text { in } \\
\text { fasting BG levels. }\end{array}$ & $\begin{array}{l}\text { Insulin not measured. Significant increase in } \\
\text { Hb. Significant increase in } \\
\text { antioxidant agents. }\end{array}$ & {$[77]$} \\
\hline $\begin{array}{l}\text { Healthy subjects } \\
\text { (Quasi-experimental study) }\end{array}$ & $\begin{array}{l}5 \text { females, } 5 \text { males; } \\
\text { age range } 20-40 \text { years }\end{array}$ & Leaf powder & $\begin{array}{l}0 \mathrm{~g} \text { (baseline), } 1 \mathrm{~g} \text { (first } \\
\text { dose after } 2 \text { weeks), } 2 \\
\mathrm{~g} \text { (second dose after } 2 \\
\text { weeks) and } 4 \mathrm{~g} \text { (third } \\
\text { dose after } 2 \text { weeks). }\end{array}$ & No significant changes. & $\begin{array}{l}\text { Significant increase in plasma insulin } 6 \mathrm{~h} \\
\text { after MO ingestion. Insulin levels with } \mathrm{MO} \\
0 \mathrm{~g}, 1 \mathrm{~g}, 2 \mathrm{~g} \text {, and } 4 \mathrm{~g} \text { were } 2.3 \pm 0.9,2.7 \pm 1.0 \\
3.3 \pm 1.4 \text {, and } 4.1 \pm 1.7 \mu \mathrm{U} / \mathrm{mL} \text {, respectively } \\
(p=0.024 \\
\text { and } p=0.011 \text { for high dose vs. baseline and } \\
\text { high dose } \\
\text { vs. low dose, respectively). No changes in } \\
\text { BUN, creatinine, ALT or AST. }\end{array}$ & [78] \\
\hline $\begin{array}{l}\text { Healthy subjects } \\
\text { (Randomized crossover } \\
\text { design study) }\end{array}$ & $\begin{array}{l}6 \text { females, } 4 \text { males; } \\
\text { age average } 22.2 \pm 0.2 \\
\text { years }\end{array}$ & Aqueous Leaf extract & $\begin{array}{l}500 \mathrm{mg} \text { on the first } \\
\text { visit and } 500 \mathrm{mg} \text { on } \\
\text { the second visit, } 2 \\
\text { weeks later. }\end{array}$ & No significant changes. & $\begin{array}{l}\text { Insulin not measured.Significant increase in } \\
\text { antioxidant capacity. Significant reduction } \\
\text { in MDA. }\end{array}$ & [79] \\
\hline $\begin{array}{l}\text { Type } 2 \mathrm{DM} \text { (Prospective } \\
\text { randomized } \\
\text { placebo-controlled study) }\end{array}$ & $\begin{array}{l}9 \text { females, } 7 \text { males; } \\
\text { age range } 20-70 \text { years }\end{array}$ & Leaf powder capsules & $\begin{array}{l}4 \mathrm{~g} \text { daily before } \\
\text { breakfast and dinner } \\
\text { for } 1 \text { month. }\end{array}$ & No significant changes. & $\begin{array}{l}\text { Insulin not measured. } \\
\text { No significant difference in } \mathrm{HbA}_{1 \mathrm{C}} \text {. } \\
\text { No changes in } \mathrm{BUN} \text {, creatinine, ALT or AST. }\end{array}$ & {$[80]$} \\
\hline $\begin{array}{l}\text { DM (type not } \\
\text { specified)(Prospective quasi } \\
\text { experimental study) }\end{array}$ & $\begin{array}{l}48 \text { females, } 12 \text { males; } \\
\text { age range } 19-65 \text { years }\end{array}$ & Leaf powder capsules & $\begin{array}{l}500 \text { mg capsule } \\
\text { ( } 3 \text { times/day) for } \\
12 \text { weeks }\end{array}$ & $\begin{array}{l}\text { Glycaemia not reported, but } \\
\text { significant reduction in HbA1c in } \\
\text { MO-treated patients. }\end{array}$ & $\begin{array}{l}\text { Insulin not measured. Significant reduction } \\
\text { in high specificity C-Reactive Protein, in } \\
\text { MO-treated patients. }\end{array}$ & [81] \\
\hline $\begin{array}{l}\text { Type } 2 \mathrm{DM} \text { and healthy } \\
\text { subjects (Randomized } \\
\text { controlled trial) }\end{array}$ & $\begin{array}{l}17 \mathrm{DM} \text { ( } 9 \text { females, } \\
8 \text { males); } 10 \text { healthy } \\
\text { (6 females, } 4 \text { males) }\end{array}$ & Leaf powder & $20 \mathrm{~g}$ once & $\begin{array}{l}\text { Significant reduction in glycaemia up } \\
\text { to } 150 \text { min after intake. } \\
\text { The mean glycemic meal response was } \\
\text { lower with } 20 \mathrm{~g} \mathrm{MO}(268 \pm 18 \mathrm{mg} / \mathrm{dL}) \\
\text { than that obtained with Con } \\
(296 \pm 17 \mathrm{mg} / \mathrm{dL}, p<0.001) \text { in diabetic } \\
\text { subjects. }\end{array}$ & $\begin{array}{l}\text { Insulin not measured. Significant reduction } \\
\text { in } \alpha \text {-amylase activity. }\end{array}$ & [82] \\
\hline
\end{tabular}


A study conducted on postmenopausal women without diabetes, showed that daily oral consumption of $7 \mathrm{~g}$ of $\mathrm{MO}$ leaf powder during three months, significantly reduced fasting blood glucose levels and increased hemoglobin values [77]. In addition, several antioxidant markers were modified in these women, reporting a significant augment in serum retinol, serum ascorbic acid, glutathione peroxidase, and SOD, whereas a significant decrease of MDA was observed. The authors of this publication mentioned no secondary or negative effects after MO ingestion. Although, the authors failed to report if background diet was either controlled or monitored, therefore, is unclear if other dietary or external factors affected the results. A study conducted on male and female healthy volunteers receiving increasing oral doses of MO leaf powder $(1 \mathrm{~g}, 2 \mathrm{~g}$, and $4 \mathrm{~g})$ every two weeks, showed no significant reduction in fasting plasma glucose, but a significant increase in insulin blood level [78]. The largest increase in insulin was found when the subjects received $4 \mathrm{~g}$ of MO leaf powder. In addition, the authors reported no changes in BUN, creatinine, alanine aminotransferase (ALT), and aspartate aminotransferase (AST) after MO ingestion. No secondary or negative effects after MO ingestion were reported. The authors failed to mention if background diet either was controlled or monitored, thus, is not clear if insulin changes observed were due to an external factor. Another study done on male and female healthy volunteers which received a single oral dose of $500 \mathrm{mg}$ MO aqueous leaf extract, showed no significant changes in the levels of fasting plasma glucose at 30,60, 90, and $120 \mathrm{~min}$ after MO ingestion [79]. However, acute changes were observed in other aspects, reporting a significant decrease in MDA levels and a significant increase in antioxidant markers. Participants were asked to fast for at least $8 \mathrm{~h}$ before MO ingestion and to maintain their typical diet, physical activity, and general lifestyle throughout the study. Furthermore, a randomized placebo controlled clinical study that compared the effect of MO leaf capsules and placebo in therapy-naïve type $2 \mathrm{DM}$ patients, found no significant differences in glycaemia or $\mathrm{HbA}_{1 \mathrm{C}}$ levels [80]. The patients received $4 \mathrm{~g}$ of $\mathrm{MO}$ leaf powder capsules twice a day, before breakfast and before dinner, over a period of four weeks. No incidence of hypoglycemia presented with MO treatment, although four patients reported transient diarrhea. As well, no significant differences were found for BUN, creatinine, ALT, and AST. The authors failed to mention if background diet either was controlled or monitored. Another study examined the effects of 12 week MO treatment (500 mg three times/day) in DM patients, finding significant plasmatic reductions in both $\mathrm{HbA}_{1 \mathrm{C}}$ levels and high specificity C-reactive protein [81], however, this study has serious limitations, which included not reporting the changes in blood glucose or insulin, no randomization or blinding in study design, not specifying the type of diabetes of participants, and having a very large age distribution, among others. Each participant had a home partner responsible for monitoring the compliance of the treatment, as well as to prevent smoking, alcoholic beverage intake, and endurance exercise a day before the blood extractions. Finally, a recent study conducted on type 2 $\mathrm{DM}$ and healthy volunteers from a Saharawi population living in refugee camps, shown a significant reduction in glycaemia and in $\alpha$-amylase activity after MO ingestion [82]. Participants were provided a meal supplemented with $20 \mathrm{~g}$ of MO leaf powder, and glycaemia was determined postprandial at 30 , $60,90,120,150$, and $180 \mathrm{~min}$. No significant differences in glycaemia were observed for the healthy subjects, while significant reductions in glycaemia were observed at 90, 120, and $150 \mathrm{~min}$ after MO treatment in individuals with diabetes. The researchers prepared a specific meal for the participants, based on $80 \mathrm{~g}$ of rice and $160 \mathrm{~g}$ of camel meat stew. Therefore, the diet was tightly controlled and homogenous between all participants. Insulin or $\mathrm{HbA}_{1 \mathrm{C}}$ levels were not reported.

Two studies (NCT03189407 and NCT02308683) involving MO and diabetes are registered in the United States National Institute of Health (NIH) clinical trials database, https://clinicaltrials.gov/. The status for both studies appears as completed but no research results are provided. Study NCT03189407 was done on type $2 \mathrm{DM}$ patients receiving metformin together with $400 \mathrm{~g}$ of $\mathrm{MO}$ in tea twice a day. This study planned to measure blood glucose levels and changes in plasmatic levels of metformin. Study NCT02308683 was done on adult DM patients, which received a 12 week supplement of MO and corresponds to the publication [81], included in Table 2. 


\section{Conclusions}

The tree MO has been proposed and studied as an antidiabetic agent. In this review, we included animal in vivo and human reports about the role MO may play in diabetes, focused on changes in glycaemia and insulin.

Ingestion or treatment with $\mathrm{MO}$ induces robust changes in glycaemia levels. All animal studies included in this review reported a significant reduction either in glycaemia or in glucose tolerance tests. Regardless of the tree part used (leaves, seeds or fruit), or the type of extract (powder, aqueous, methanolic or ethanolic), all diabetes animal models showed a significant reduction of glycaemia in diabetic animals treated with $\mathrm{MO}$ as compared with diabetic controls. The evidence for acute antihyperglycemic effects of MO extract on diabetic animal models seems to be robust, but more chronic and long-term studies are needed. This is important, as DM and metabolic syndrome have a chronic nature and novel therapeutic (or complementary) agents need to take into consideration this aspect. An important limitation regarding the studies included in this review is related to the wide variation in the parameters reported. In particular, the absence of other supporting measurements of glucose metabolism, such as glycosylated hemoglobin or glucagon, limits the conclusions regarding the effectiveness of MO treatment in animal models of diabetes. Therefore, future research should examine not only hypoglycemic effects of $\mathrm{MO}$, but also additional parameters related to glucose function.

Opposed to animal studies, only two studies in humans, one conducted on postmenopausal women and one in type $2 \mathrm{DM}$, showed a significant glycaemia reduction, the other four, two with healthy subjects and two in patients with DM, failed to show a significant reduction. These results are difficult to compare as all studies had different methodologies and used a diverse range of doses. For example, the study on postmenopausal women used the highest amount of MO and for a longer period ( $7 \mathrm{~g}$ daily for three months) as compared with the other five studies. Similar to healthy human subjects, many animal studies failed to observe a significant decrease in glycaemia after MO use in nondiabetic controls, suggesting that glucose regulation may be more effective under pathological conditions. A more precise isolation and characterization of the various phytochemicals present in MO may be of help determining which ones (or a combination of) produce acute or chronic glycaemia lowering effects. Nevertheless, the evidence indicates that the ingestion of MO is safe both in animals (between 50 and $700 \mathrm{mg} / \mathrm{kg}$ ) and in humans (between $500 \mathrm{mg}$ to $7 \mathrm{~g}$ ). This safety profile suggests the design of nutritional investigations including $\mathrm{MO}$ as a supplement or complement for patients suffering from metabolic or even neurological diseases. Despite the safety profile of MO, a possible limitation in human studies, in particular long-lasting trials, may be related to the taste of $\mathrm{MO}$, as poor taste acceptability of this tree in meals has been reported [82]. In addition, most studies used MO leaves, and only a few were done with seeds or fruits. Therefore, more comparative studies between the different parts of the tree are also suggested in order to determine which tree part (or a mix of them) is more efficient.

Evidence regarding changes in insulin levels due to MO intervention is not as robust as that for antihyperglycemic effects. Some works showed a significant increase in insulin levels, but many other studies on rats and mice failed to replicate this. Similar findings occurred in human studies, where only one (in healthy subjects) reported insulin, showing a significant increase in insulin levels in individuals treated with MO. Therefore, more research is needed to clarify if MO has an effect on insulin levels or activity. This is important when determining the type of DM on which intervention is planned, as type I would benefit from insulin increase, and type II, due to hyperinsulinemia, may not. Other effects commonly reported in the animal models and in human studies are in line with in vitro observations such as strong antioxidant and lipid metabolic regulating properties, which could also be of benefit for the treatment of various metabolic and neurological conditions, including diabetes.

Author Contributions: Conceptualization, K.V.-S. and R.E.G.-R.; writing-original draft preparation E.G.-J., K.V.-S., and R.E.G.-R.; writing-review and editing, E.G.-J., K.V.-S., and R.E.G.-R.; supervision, K.V.-S. and R.E.G.-R; project administration K.V.-S. and R.E.G.-R. 
Funding: This research received no external funding.

Conflicts of Interest: The authors declare no conflict of interest.

\section{References}

1. Leone, A.; Spada, A.; Battezzati, A.; Schiraldi, A.; Aristil, J.; Bertoli, S. Cultivation, genetic, ethnopharmacology, phytochemistry and pharmacology of Moringa oleifera Leaves: An overview. Int. J. Mol. Sci. 2015, 16, 12791-12835. [CrossRef]

2. Popoola, J.O.; Obembe, O.O. Local knowledge, use pattern and geographical distribution of Moringa oleifera Lam. (Moringaceae) in Nigeria. J. Ethnopharmacol. 2013, 150, 682-691. [CrossRef] [PubMed]

3. Falowo, A.B.; Mukumbo, F.E.; Idamokoro, E.M.; Lorenzo, J.M.; Afolayan, A.J.; Muchenje, V. Multi-functional application of Moringa oleifera Lam. in nutrition and animal food products: A review. Food Res. Int. 2018, 106, 317-334. [CrossRef] [PubMed]

4. Anwar, F.; Latif, S.; Ashraf, M.; Gilani, A.H. Moringa oleifera: A food plant with multiple medicinal uses. Phyther. Res. 2007, 21, 17-25. [CrossRef] [PubMed]

5. Jaja-Chimedza, A.; Graf, B.L.; Simmler, C.; Kim, Y.; Kuhn, P.; Pauli, G.F.; Raskin, I. Biochemical characterization and anti-inflammatory properties of an isothiocyanate-enriched moringa (Moringa oleifera) seed extract. PLoS ONE 2017, 12, e0182658. [CrossRef] [PubMed]

6. Samson, S.L.; Garber, A.J. Metabolic Syndrome. Endocrinol. Metab. Clin. N. Am. 2014, 43, 1-23. [CrossRef]

7. González-Reyes, R.E.; Aliev, G.; Ávila-Rodrigues, M.; Barreto, G.E. Alterations in glucose metabolism on cognition: A possible link between diabetes and dementia. Curr. Pharm. Des. 2016, 22, 812-818. [CrossRef]

8. Feldman, E.L.; Nave, K.; Jensen, T.S.; Bennett, D.L.H. New Horizons in Diabetic Neuropathy: Mechanisms, Bioenergetics, and Pain. Neuron 2017, 93, 1296-1313. [CrossRef]

9. Azad, S.B.; Ansari, P.; Azam, S.; Hossain, S.M.; Shahid, M.I.; Hasan, M.; Hannan, J.M.A. Anti-hyperglycaemic activity of Moringa oleifera is partly mediated by carbohydrase inhibition and glucose-fibre binding. Biosci. Rep. 2017, 37, BSR20170059. [CrossRef]

10. Abd El Latif, A.; El Bialy, B.E.S.; Mahboub, H.D.; Abd Eldaim, M.A. Moringa oleifera leaf extract ameliorates alloxan-induced diabetes in rats by regeneration of $\beta$ cells and reduction of pyruvate carboxylase expression. Biochem. Cell Biol. 2014, 92, 413-419. [CrossRef]

11. Igado, O.O.; Olopade, J.O. A Review on the possible neuroprotective effects of Moringa oleifera leaf extract. Niger. J. Physiol. Sci. 2017, 31, 183-187. [PubMed]

12. Leone, A.; Spada, A.; Battezzati, A.; Schiraldi, A.; Aristil, J.; Bertoli, S. Moringa oleifera seeds and oil: Characteristics and uses for human health. Int. J. Mol. Sci. 2016, 17, 2141. [CrossRef] [PubMed]

13. Guevara, A.P.; Vargas, C.; Sakurai, H.; Fujiwara, Y.; Hashimoto, K.; Maoka, T.; Kozuka, M.; Ito, Y.; Tokuda, H.; Nishino, H. An antitumor promoter from Moringa oleifera Lam. Mutat. Res. 1999, 440, 181-188. [CrossRef]

14. Nadeem, M.; Imran, M. Promising features of Moringa oleifera oil: Recent updates and perspectives. Lipids Health Dis. 2016, 15, 212. [CrossRef]

15. Mehta, K.; Balaraman, R.; Amin, A.H.; Bafna, P.A.; Gulati, O.D. Effect of fruits of Moringa oleifera on the lipid profile of normal and hypercholesterolaemic rabbits. J. Ethnopharmacol. 2003, 86, 191-195. [CrossRef]

16. Kumar, N.A.; Pari, L. Antioxidant action of Moringa oleifera Lam. (Drumstick) against antitubercular drugs induced lipid peroxidation in rats. J. Med. Food 2003, 6, 255-259. [CrossRef]

17. Olson, M.E.; Sankaran, R.P.; Fahey, J.W.; Grusak, M.A.; Odee, D.; Nouman, W. Leaf protein and mineral concentrations across the "Miracle Tree" genus Moringa. PLoS ONE 2016, 11, e0159782. [CrossRef]

18. Kou, X.; Li, B.; Olayanju, J.B.; Drake, J.M.; Chen, N. Nutraceutical or pharmacological potential of Moringa oleifera Lam. Nutrients 2018, 10, 343. [CrossRef]

19. Mahdi, J.H.; Khan, N.K.A.; Asmawi, M.Z.B.; Mahmud, R.; A/L Murugaiyah, V. In vivo anti-arthritic and anti-nociceptive effects of ethanol extract of Moringa oleifera leaves on complete Freund's adjuvant (CFA)-induced arthritis in rats. Integr. Med. Res. 2018, 7, 85-94. [CrossRef]

20. He, T.; Huang, Y.; Huang, Y.; Wang, X.; Hu, J.; Sheng, J. Structural elucidation and antioxidant activity of an arabinogalactan from the leaves of Moringa oleifera. Int. J. Biol. Macromol. 2018, 112, 126-133. [CrossRef]

21. Feustel, S.; Ayón-Pérez, F.; Sandoval-Rodriguez, A.; Rodríguez-Echevarría, R.; Contreras-Salinas, H.; Armendáriz-Borunda, J.; Sánchez-Orozco, L. Protective effects of Moringa oleifera on HBV genotypes C and H transiently transfected Huh7 cells. J. Immunol. Res. 2017, 2017, 6063850. [CrossRef] [PubMed] 
22. Cáceres, A.; Cabrera, O.; Morales, O.; Mollinedo, P.; Mendia, P. Pharmacological properties of Moringa oleifera. 1: Preliminary screening for antimicrobial activity. J. Ethnopharmacol. 1991, 33, 213-216. [CrossRef]

23. Vergara-Jimenez, M.; Almatrafi, M.M.; Fernandez, M.L. Bioactive components in Moringa oleifera leaves protect against chronic disease. Antioxidants 2017, 6, 91. [CrossRef] [PubMed]

24. Igwilo, I.O.; Ezeonu, F.C.; Ezekwesili-Ofili, J.O.; Igwilo, S.; Nsofor, C.; Abdulsalami, M.; Obi, E. Anti-nutritional factors in the roots of a local cultivar of Moringa oleifera (Lam). Pak. J. Biol. Sci. 2014, 17, 114-117. [CrossRef]

25. Choudhary, M.K.; Bodakhe, S.H.; Gupta, S.K. Assessment of the antiulcer potential of Moringa oleifera root-bark extract in rats. J. Acupunct. Meridian Stud. 2013, 6, 214-220. [CrossRef]

26. Singh, B.N.; Singh, B.R.; Singh, R.L.; Prakash, D.; Dhakarey, R.; Upadhyay, G.; Singh, H.B. Oxidative DNA damage protective activity, antioxidant and anti-quorum sensing potentials of Moringa oleifera. Food Chem. Toxicol. 2009, 47, 1109-1116. [CrossRef]

27. Ndong, M.; Uehara, M.; Katsumata, S.; Suzuki, K. Effects of oral administration of Moringa oleifera Lam on glucose tolerance in Goto-Kakizaki and Wistar rats. J. Clin. Biochem. Nutr. 2007, 40, 229-233. [CrossRef]

28. Kellett, G.L.; Brot-Laroche, E. Apical GLUT2: A major pathway of intestinal sugar absorption. Diabetes 2005, 54, 3056-3062. [CrossRef]

29. Dyer, J.; Wood, I.S.; Palejwala, A.; Ellis, A.; Shirazi-Beechey, S.P. Expression of monosaccharide transporters in intestine of diabetic humans. Am. J. Physiol. Gastrointest. Liver Physiol. 2002, 282, G241-G248. [CrossRef]

30. Meneses, M.J.; Silva, B.M.; Sousa, M.; Sá, R.; Oliveira, P.F.; Alves, M.G. Antidiabetic drugs: Mechanisms of action and potential outcomes on cellular metabolism. Curr. Pharm. Des. 2015, 21, 3606-3620. [CrossRef]

31. Kwon, O.; Eck, P.; Chen, S.; Corpe, C.P.; Lee, J.; Kruhlak, M.; Levine, M. Inhibition of the intestinal glucose transporter GLUT2 by flavonoids. FASEB J. 2007, 21, 366-377. [CrossRef] [PubMed]

32. Song, J.; Kwon, O.; Chen, S.; Daruwala, R.; Eck, P.; Park, J.B.; Levine, M. Flavonoid Inhibition of Sodium-dependent Vitamin C Transporter 1 (SVCT1) and Glucose Transporter Isoform 2 (GLUT2), Intestinal Transporters for Vitamin C and Glucose. J. Biol. Chem. 2002, 277, 15252-15260. [CrossRef] [PubMed]

33. Eid, H.M.; Nachar, A.; Thong, F.; Sweeney, G.; Haddad, P.S. The molecular basis of the antidiabetic action of quercetin in cultured skeletal muscle cells and hepatocytes. Pharmacogn. Mag. 2015, 11, 74-81. [PubMed]

34. Adisakwattana, S.; Chanathong, B. Alpha-glucosidase inhibitory activity and lipid-lowering mechanisms of Moringa oleifera leaf extract. Eur. Rev. Med. Pharmacol. Sci. 2011, 15, 803-808.

35. Tadera, K.; Minami, Y.; Takamatsu, K.; Matsuoka, T. Inhibition of alpha-glucosidase and alpha-amylase by flavonoids. J. Nutr. Sci. Vitaminol. 2006, 52, 149-153. [CrossRef]

36. Youl, E.; Bardy, G.; Magous, R.; Cros, G.; Sejalon, F.; Virsolvy, A.; Richard, S.; Quignard, J.; Gross, R.; Petit, P.; et al. Quercetin potentiates insulin secretion and protects INS-1 pancreatic b-cells against oxidative damage via the ERK1/2. Br. J. Pharmacol. 2010, 161, 799-814. [CrossRef]

37. Ríos, J.L.; Francini, F.; Schinella, G.R. Natural products for the treatment of Type 2 Diabetes Mellitus. Planta Med. 2015, 81, 975-994. [CrossRef]

38. Pitschmann, A.; Zehl, M.; Atanasov, A.G.; Dirsch, V.M.; Heiss, E.; Glasl, S. Walnut leaf extract inhibits PTP1B and enhances glucose-uptake in vitro. J. Ethnopharmacol. 2014, 152, 599-602. [CrossRef]

39. Vessal, M.; Hemmati, M.; Vasei, M. Antidiabetic effects of quercetin in streptozocin-induced diabetic rats. Comp. Biochem. Physiol. C Toxicol. Pharmacol. 2003, 135, 357-364. [CrossRef]

40. Mbikay, M. Therapeutic potential of Moringa oleifera leaves in chronic hyperglycemia and dyslipidemia: A review. Front. Pharmacol. 2012, 3, 24. [CrossRef]

41. Wolff, S.P.; Jiang, Z.Y.; Hunt, J.V. Protein glycation and oxidative stress in diabetes mellitus and ageing. Free Radic. Biol. Med. 1991, 10, 339-352. [CrossRef]

42. Nunthanawanich, P.; Sompong, W.; Sirikwanpong, S.; Mäkynen, K.; Adisakwattana, S.; Dahlan, W.; Ngamukote, S. Moringa oleifera aqueous leaf extract inhibits reducing monosaccharide-induced protein glycation and oxidation of bovine serum albumin. Springerplus 2016, 5, 1098. [CrossRef] [PubMed]

43. Sadowska-Bartosz, I.; Galiniak, S.; Bartosz, G. Kinetics of glycoxidation of bovine serum albumin by methylglyoxal and glyoxal and its prevention by various compounds. Molecules 2014, 19, 4880-4896. [CrossRef] [PubMed]

44. Verma, A.R.; Vijayakumar, M.; Mathela, C.S.; Rao, C.V. In vitro and in vivo antioxidant properties of different fractions of Moringa oleifera leaves. Food Chem. Toxicol. 2009, 47, 2196-2201. [CrossRef] [PubMed] 
45. Sangkitikomol, W.; Rocejanasaroj, A.; Tencomnao, T. Effect of Moringa oleifera on advanced glycation end-product formation and lipid metabolism gene expression in HepG2 cells. Genet. Mol. Res. 2014, 13, 723-735. [CrossRef]

46. Moyo, B.; Oyedemi, S.; Masika, P.J.; Muchenje, V. Polyphenolic content and antioxidant properties of Moringa oleifera leaf extracts and enzymatic activity of liver from goats supplemented with Moringa oleifera leaves/sunflower seed cake. Meat Sci. 2012, 91, 441-447. [CrossRef]

47. Sosa-Gutiérrez, J.A.; Valdéz-Solana, M.A.; Forbes-Hernández, T.Y.; Avitia-Domínguez, C.I.; Garcia-Vargas, G.G.; Salas-Pacheco, J.M.; Flores-Herrera, O.; Téllez-Valencia, A.; Battino, M.; Sierra-Campos, E. Effects of Moringa oleifera leaves extract on high glucose-induced metabolic changes in HepG2 cells. Biology (Basel) 2018, 7, 37. [CrossRef]

48. Chumark, P.; Khunawat, P.; Sanvarinda, Y.; Phornchirasilp, S.; Morales, N.P.; Phivthong-Ngam, L.; Ratanachammong, P.; Srisawat, S.; Pongrapeeporn, K.U. The in vitro and ex vivo antioxidant properties, hypolipidaemic and antiatherosclerotic activities of water extract of Moringa oleifera Lam. leaves. J. Ethnopharmacol. 2008, 116, 439-446. [CrossRef]

49. Alhakmani, F.; Kumar, S.; Khan, S.A. Estimation of total phenolic content, in-vitro antioxidant and anti-inflammatory activity of flowers of Moringa oleifera. Asian Pac. J. Trop. Biomed. 2013, 3, 623-627. [CrossRef]

50. Giacoppo, S.; Rajan, T.S.; De Nicola, G.R.; Iori, R.; Rollin, P.; Bramanti, P.; Mazzon, E. The isothiocyanate isolated from Moringa oleifera shows potent anti-inflammatory activity in the treatment of murine subacute Parkinson's disease. Rejuvenation Res. 2017, 20, 50-63. [CrossRef]

51. Xu, Y.-B.; Chen, G.-L.; Guo, M.-Q. Antioxidant and anti-inflammatory activities of the crude extracts of Moringa oleifera from Kenya and their correlations with flavonoids. Antioxidants 2019, 8, 296. [CrossRef] [PubMed]

52. Park, E.J.; Cheenpracha, S.; Chang, L.C.; Kondratyuk, T.P.; Pezzuto, J.M. Inhibition of lipopolysaccharide- induced cyclooxygenase-2 and inducible nitric oxide synthase expression by 4-[(2'-O-acetyl- $\alpha$-L-rhamnosyloxy)benzyl]isothiocyanate from Moringa oleifera. Nutr. Cancer 2012, 63, 971-982. [CrossRef] [PubMed]

53. Lee, H.-J.; Jeong, Y.-J.; Lee, T.-S.; Park, Y.-Y.; Chae, W.-G.; Chung, I.-K.; Chang, H.-W.; Kim, C.-H.; Choi, Y.-H.; Kim, W.-J.; et al. Moringa fruit inhibits LPS-induced NO/iNOS expression through suppressing the NF- $\mathrm{kB}$ activation in RAW264.7 cells. Am. J. Chin. Med. 2013, 41, 1109-1123. [CrossRef] [PubMed]

54. Zhang, Y.; Liu, D. Flavonol kaempferol improves chronic hyperglycemia-impaired pancreatic beta-cell viability and insulin secretory function. Eur. J. Pharmacol. 2011, 670, 325-332. [CrossRef]

55. Randriamboavonjy, J.I.; Loirand, G.; Vaillant, N.; Lauzier, B.; Derbré, S.; Michalet, S.; Pacaud, P.; Tesse, A. Cardiac protective effects of Moringa oleifera seeds in spontaneous hypertensive rats. Am. J. Hypertens. 2016, 29, 873-881. [CrossRef]

56. Botta, M.; Audano, M.; Sahebkar, A.; Sirtori, C.R.; Mitro, N.; Ruscica, M. PPAR agonists and metabolic syndrome: An established role? Int. J. Mol. Sci. 2018, 19, 1197. [CrossRef]

57. Villaruel-López, A.; López-de la Mora, D.; Vasquez-Paulino, O.; Puebla-Mora, A.; Torres-vitela, M.; Guerrero-Quiroz, L.; Nuño, K. Effect of Moringa oleifera consumption on diabetic rats. BMC Complement. Altern. Med. 2018, 18, 127. [CrossRef]

58. López, M.; Ríos-Silva, M.; Huerta, M.; Cárdenas, Y.; Bricio-Barrios, J.A.; Díaz-Reval, M.I.; Urzúa, Z.; Huerta-Trujillo, M.; López-Quesada, K.; Trujillo, X. Effects of Moringa oleifera leaf powder on metabolic syndrome induced in male Wistar rats: A preliminary study. J. Int. Med. Res. 2018, 46, 3327-3336. [CrossRef]

59. Paula, P.C.; Sousa, D.O.; Oliveira, J.T.; Carvalho, A.F.; Alves, B.G.; Pereira, M.L.; Farias, D.F.; Viana, M.P.; Santos, F.A.; Morais, T.C.; et al. A protein isolate from Moringa oleifera leaves has hypoglycemic and antioxidant effects in alloxan-induced diabetic mice. Molecules 2017, 22, 271. [CrossRef]

60. Jaiswal, D.; Rai, P.K.; Kumar, A.; Mehta, S.; Watal, G. Effect of Moringa oleifera Lam. leaves aqueous extract therapy on hyperglycemic rats. J. Ethnopharmacol. 2009, 123, 392-396. [CrossRef]

61. Yassa, H.D.; Tohamy, A.F. Extract of Moringa oleifera leaves ameliorates streptozotocin-induced Diabetes mellitus in adult rats. Acta Histochem. 2014, 116, 844-854. [CrossRef] [PubMed]

62. Abd Eldaim, M.A.; Shaban Abd Elrasoul, A.; Abd Elaziz, S.A. An aqueous extract from Moringa oleifera leaves ameliorates hepatotoxicity in alloxan-induced diabetic rats. Biochem. Cell Biol. 2017, 95, 524-530. [CrossRef] [PubMed] 
63. Khan, W.; Parveen, R.; Chester, K.; Parveen, S.; Ahmad, S. Hypoglycemic potential of aqueous extract of Moringa oleifera leaf and in vivo GC-MS metabolomics. Front. Pharmacol. 2017, 8, 577. [CrossRef] [PubMed]

64. Olayaki, L.A.; Irekpita, J.E.; Yakubu, M.T.; Ojo, O.O. Methanolic extract of Moringa oleifera leaves improves glucose tolerance, glycogen synthesis and lipid metabolism in alloxan-induced diabetic rats. J. Basic Clin. Physiol. Pharmacol. 2015, 26, 583-593. [CrossRef]

65. Omodanisi, E.I.; Aboua, Y.G.; Chegou, N.N.; Oguntibeju, O.O. Hepatoprotective, Antihyperlipidemic, and Anti-inflammatory Activity of Moringa oleifera in Diabetic-induced Damage in Male Wistar Rats. Pharmacogn. Res. 2017, 9, 182-187.

66. Sánchez-Muñoz, M.A.; Valdez-Solana, M.A.; Campos-Almazán, M.I.; Flores-Herrera, O.; Esparza-Perusquía, M.; Olvera-Sánchez, S.; García-Arenas, G.; Avitia-Domínguez, C.; Téllez-Valencia, A.; Sierra-Campos, E. Streptozotocin-Induced Adaptive Modification of Mitochondrial Supercomplexes in Liver of Wistar Rats and the Protective Effect of Moringa oleifera Lam. Biochem. Res. Int. 2018, 2018, 5681081.

67. Olurishe, C.; Kwanashie, H.; Zezi, A.; Danjuma, N.; Mohammed, B. Chronic administration of ethanol leaf extract of Moringa oleifera Lam. (Moringaceae) may compromise glycaemic efficacy of Sitagliptin with no significant effect in retinopathy in a diabetic rat model. J. Ethnopharmacol. 2016, 194, 895-903. [CrossRef]

68. Tang, Y.; Choi, E.J.; Han, W.C.; Oh, M.; Kim, J.; Hwang, J.-Y.; Park, P.-J.; Moon, S.-H.; Kim, Y.-S.; Kim, E.-K. Moringa oleifera from Cambodia ameliorates oxidative stress, hyperglycemia, and kidney dysfunction in Type 2 diabetic mice. J. Med. Food 2017, 20, 502-510. [CrossRef]

69. Al-Malki, A.L.; El Rabey, H.A. The antidiabetic effect of low doses of Moringa oleifera Lam. seeds on streptozotocin induced diabetes and diabetic nephropathy in male rats. BioMed Res. Int. 2015, 2015, 381040. [CrossRef]

70. Jaja-Chimedza, A.; Zhang, L.; Wol, K.; Graf, B.L.; Kuhn, P.; Moskal, K.; Carmouche, R.; Newman, S.; Salbaum, J.M.; Raskin, I. A dietary isothiocyanate-enriched moringa (Moringa oleifera) seed extract improves glucose tolerance in a high-fat-diet mouse model and modulates the gut microbiome. J. Funct. Foods 2018, 47, 376-385. [CrossRef]

71. Wang, F.; Zhong, H.; Chen, W.; Liu, Q.; Li, C. Potential hypoglycaemic activity phenolic glycosides from Moringa oleifera seeds. Nat. Prod. Res. 2017, 31, 1869-1874. [CrossRef] [PubMed]

72. Rafaat, K.; Hdaib, F. Neuroprotective effects of Moringa oleifera: Bio-guided GC-MS identification of active compounds in diabetic neuropathic pain model. Chin. J. Integr. Med. 2017. [CrossRef] [PubMed]

73. Gupta, R.; Mathur, M.; Bajaj, V.K.; Katariya, P.; Yadav, S.; Kamal, R.; Gupta, R.S. Evaluation of antidiabetic and antioxidant activity of Moringa oleifera in experimental diabetes. J. Diabetes 2012, 4, 164-171. [CrossRef] [PubMed]

74. Olurishe, C.O.; Kwanashie, H.O.; Zezi, A.U.; Danjuma, N.M.; Mohammed, B. Sitagliptin-Moringa oleifera coadministration did not delay the progression nor ameliorated functional and morphological anomalies in alloxan-induced diabetic nephropathy. Indian J. Pharmacol. 2017, 49, 366-373.

75. Cazarolli, L.H.; Zanatta, L.; Jorge, A.P.; De Sousa, E.; Horst, H.; Woehl, V.M.; Pizzolatti, M.G.; Szpoganicz, B.; Silva, F.R. Follow-up studies on glycosylated flavonoids and their complexes with vanadium: Their anti-hyperglycemic potential role in diabetes. Chem. Biol. Interact. 2006, 163, 177-191. [CrossRef]

76. Amelia, D.; Santoso, B.; Purwanto, B.; Miftahussurur, M.; Joewono, H.T.; Budiono. Effects of Moringa oleifera on insulin levels and folliculogenesis in polycystic ovary syndrome model with insulin resistance. Immunol. Endocr. Metab. Agents Med. Chem. 2018, 18, 22-30. [CrossRef]

77. Kushwaha, S.; Chawla, P.; Kochhar, A. Effect of supplementation of drumstick (Moringa oleifera) and amaranth (Amaranthus tricolor) leaves powder on antioxidant profile and oxidative status among postmenopausal women. J. Food Sci. Technol. 2014, 51, 3464-3469. [CrossRef]

78. Anthanont, P.; Lumlerdkij, N.; Akarasereenont, P.; Vannasaeng, S.; Sriwijitkamol, A. Moringa Oleifera leaf increases insulin secretion after single dose administration: A preliminary study in healthy subjects. J. Med. Assoc. Thail. 2016, 99, 308-313.

79. Ngamukote, S.; Khannongpho, T.; Siriwatanapaiboon, M.; Dahlan, W.; Adisakwattana, S. Moringa Oleifera leaf extract increases plasma antioxidant status associated with reduced plasma malondialdehyde concentration without hypoglycemia in fasting healthy volunteers. Chin. J. Integr. Med. 2016, 1-6. [CrossRef]

80. Taweerutchana, R.; Lumlerdkij, N.; Vannasaeng, S.; Akarasereenont, P.; Sriwijitkamol, A. Effect of Moringa oleifera leaf capsules on glycemic control in therapy-naive Type 2 diabetes patients: A randomized placebo controlled study. Evid. Based Complement. Altern. Med. 2017, 2017, 6581390. [CrossRef] 
81. Mozo, R.N.; Caole-Ang, I. The effects of Malunggay (Moringa oleifera) leaves capsule supplements on high specificity C-reactive protein and hemoglobin A1c levels of diabetic patients in Ospital ng Maynila Medical Center: A prospective cohort study. Philipp. J. Intern. Med. 2015, 53, 1-10.

82. Leone, A.; Bertoli, S.; Di Lello, S.; Bassoli, A.; Ravasenghi, S.; Borgonovo, G.; Forlani, F.; Battezzati, A. Effect of Moringa oleifera leaf powder on postprandial blood glucose response: In vivo study on Saharawi people living in refugee camps. Nutrients 2018, 10, 1494. [CrossRef] [PubMed]

(c)

(C) 2019 by the authors. Licensee MDPI, Basel, Switzerland. This article is an open access article distributed under the terms and conditions of the Creative Commons Attribution (CC BY) license (http://creativecommons.org/licenses/by/4.0/). 